\title{
Using zebrafish larval models to study brain injury, locomotor and neuroinflammatory outcomes following intracerebral
}

\section{haemorrhage [version 1; peer review: 1 approved, 1 approved}

\section{with reservations]}

\author{
Siobhan Crilly (D1), Alexandra Njegic (D2), Sarah E. Laurie1, Elisavet Fotiou², \\ Georgina Hudson ${ }^{1}$, Jack Barrington ${ }^{1}$, Kirsty Webb², Helen L. Young 3 , \\ Andrew P. Badrock ${ }^{4}$, Adam Hurlstone ${ }^{3}$, Jack Rivers-Auty ${ }^{1}$, Adrian R. Parry-Jones², \\ Stuart M. Allan (D1*, Paul R. Kasher (D)1* \\ ${ }^{1}$ Division of Neuroscience and Experimental Psychology, School of Biological Sciences, Faculty of Biology, Medicine and Health, \\ Manchester Academic Health Science Centre, The University of Manchester, Oxford Road, Manchester, M13 9PT, UK \\ ${ }^{2}$ Division of Cardiovascular Sciences, School of Medical Sciences, Faculty of Biology, Medicine and Health, Manchester Academic \\ Health Science Centre, The University of Manchester, Oxford Road, Manchester, M13 9PT, UK \\ ${ }^{3}$ Division of Cancer Sciences, School of Medical Sciences, Faculty of Biology, Medicine and Health, Manchester Academic Health \\ Science Centre, The University of Manchester, Oxford Road, Manchester, M13 9PT, UK \\ ${ }^{4}$ Division of Evolution and Genomic Sciences, School of Biological Sciences, Faculty of Biology, Medicine and Health, Manchester \\ Academic Health Science Centre, The University of Manchester, Oxford Road, Manchester, M13 9PT, UK \\ * Equal contributors
}

V1 First published: 08 Oct 2018, 7:1617

https://doi.org/10.12688/f1000research.16473.1

Latest published: 08 Nov 2018, 7:1617

https://doi.org/10.12688/f1000research.16473.2
Open Peer Review

Approval Status

1

2

version 2

(revision)

08 Nov 2018

version 1

08 Oct 2018

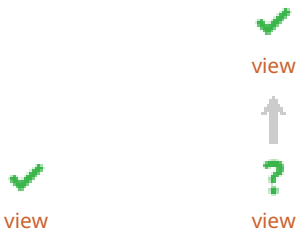

1. Claire L. Gibson (D), University of Nottingham, Nottingham, UK

2. Marietta Zille ID, University of Lübeck, Institute for Experimental and Clinical Pharmacology and Toxicology, Lübeck, 
function and neuroinflammation following ICH. We show that ICH in both zebrafish larval models is comparable in timing, frequency and location. ICH results in increased brain cell death and a persistent locomotor deficit. Additionally, in haemorrhaged larvae we observed a significant increase in macrophage recruitment to the site of injury. Live in vivo imaging allowed us to track active macrophage-based phagocytosis of dying brain cells 24 hours after haemorrhage. Morphological analyses and quantification indicated that an increase in overall macrophage activation occurs in the haemorrhaged brain. Our study shows that in zebrafish larvae, bleeding in the brain induces quantifiable phenotypic outcomes that mimic key features of human $\mathrm{ICH}$. We hope that this methodology will enable the pre-clinical ICH community to adopt the zebrafish larval model as an alternative to rodents, supporting future high throughput drug screening and as a complementary approach to elucidating crucial mechanisms associated with ICH pathophysiology.

\section{Keywords}

Intracerebral haemorrhage, zebrafish, neuroinflammation, animal models, pre-clinical

\section{Germany}

Fraunhofer Research Institution for Marine

Biotechnology and Cell Technology, Lübeck,

Germany

Any reports and responses or comments on the article can be found at the end of the article.

\section{$\mathrm{NC}$ $3 R^{s}$}

This article is included in the NC3Rs gateway.

Corresponding author: Paul R. Kasher (paul.kasher@manchester.ac.uk)

Author roles: Crilly S: Conceptualization, Data Curation, Formal Analysis, Investigation, Methodology, Project Administration, Software, Validation, Visualization, Writing - Original Draft Preparation, Writing - Review \& Editing; Njegic A: Formal Analysis, Investigation, Methodology, Validation, Writing - Review \& Editing; Laurie SE: Formal Analysis, Investigation, Validation, Writing - Review \& Editing; Fotiou E: Formal Analysis, Investigation, Writing - Review \& Editing; Hudson G: Investigation, Methodology, Writing - Review \& Editing; Barrington J: Formal Analysis, Writing - Review \& Editing; Webb K: Investigation, Methodology, Writing - Review \& Editing; Young HL: Methodology, Validation, Writing - Review \& Editing; Badrock AP: Methodology, Validation, Writing - Review \& Editing; Hurlstone A: Resources, Writing - Review \& Editing; Rivers-Auty J: Data Curation, Formal Analysis, Software, Writing - Review \& Editing; Parry-Jones AR: Conceptualization, Funding Acquisition, Project Administration, Supervision, Writing - Review \& Editing; Allan SM: Conceptualization, Funding Acquisition, Project Administration, Resources, Supervision, Writing - Review \& Editing; Kasher PR: Conceptualization, Formal Analysis, Funding Acquisition, Investigation, Methodology, Project Administration, Resources, Supervision, Validation, Visualization, Writing - Original Draft Preparation, Writing - Review \& Editing

Competing interests: No competing interests were disclosed.

Grant information: This study was supported by the NC3Rs (NC/N002598/1), Stroke Association (TSA LECT 2017/02), ERA-NET NEURON (MR/M501803/1) and The British Heart Foundation (FS/14/64/31291, FS/15/67/32038). We are also particularly thankful to The Natalie Kate Moss Trust for their continued financial support.

Copyright: $\odot 2018$ Crilly S et al. This is an open access article distributed under the terms of the Creative Commons Attribution License, which permits unrestricted use, distribution, and reproduction in any medium, provided the original work is properly cited. Data associated with the article are available under the terms of the Creative Commons Zero "No rights reserved" data waiver (CCO 1.0 Public domain dedication).

How to cite this article: Crilly S, Njegic A, Laurie SE et al. Using zebrafish larval models to study brain injury, locomotor and neuroinflammatory outcomes following intracerebral haemorrhage [version 1; peer review: 1 approved, 1 approved with reservations] F1000Research 2018, 7:1617 https://doi.org/10.12688/f1000research.16473.1

First published: 08 Oct 2018, 7:1617 https://doi.org/10.12688/f1000research.16473.1 


\section{Research highlights}

\section{Scientific benefits:}

- No surgery is required so the models more accurately recapitulate spontaneous $\mathrm{ICH}$

- Ease of genetic manipulation associated with zebrafish allows for the use of transgenic and mutant lines

- Transparency of zebrafish larvae allows for non-invasive monitoring of cellular processes over time (live imaging)

\section{Rs benefits:}

- Unprotected zebrafish larvae (before $5 \mathrm{dpf}$ ) can be used as an alternative to rodent models in pre-clinical $\mathrm{ICH}$ research

\section{Practical benefits:}

- High fecundity of zebrafish pairings means that a large sample size of genetically comparable siblings can be produced easily

- Zebrafish are a lower cost organism to host than rodents

- Experimental timeline is less time consuming than typical rodent studies which look at long term outcomes of stroke commonly up to 3 months

Current applications:

- Elucidating immediate pathological outcomes of spontaneous $\mathrm{ICH}$ in the brain of intact animals

Potential applications:

- High throughput drug screens for $\mathrm{ICH}$ therapies

- Replacing the current rodent models for analysis of immediate $\mathrm{ICH}$ pathology

\section{Introduction}

Intracerebral haemorrhage (ICH) accounts for $10-15 \%$ of strokes and has the worst stroke outcomes, with a 1-month case fatality of $40 \%$ and disability in most survivors (An et al., 2017). The effects of ICH in the brain are biphasic. Primary injury following an ICH event arises due to an influx of blood into the brain and haematoma expansion which increases intracranial pressure on cerebral structures causing neuronal death and cell necrosis (Mracsko \& Veltkamp, 2014; Xi et al., 2006). A secondary wave of injury is induced by the breakdown of blood compounds which activates the immune system, further exacerbating cellular damage and death in the brain parenchyma and induces a breakdown in blood-brain barrier integrity (Lok et al., 2011). This inflammatory component is considered a viable therapeutic target in $\mathrm{ICH}$ and other forms of stroke (Veltkamp \& Gill, 2016) and targeting the toxic insult of blood components after bleed onset is being investigated clinically (Yeatts et al., 2013). However at present, apart from acute and chronic blood pressure lowering, we have no specific treatments to prevent ICH or improve patient outcomes once bleeding has occurred.

Despite representing a significant public health burden (WHO, 2017), an understanding of the fundamental pathogenesis of ICH is still lacking. Pre-clinical studies to-date have depended heavily on rodent models of $\mathrm{ICH}$, which have improved our knowledge of the basic mechanisms associated with the disease (Casals et al., 2011). However, current rodent models of ICH involve severe surgical intervention, poorly recapitulating the spontaneous and immediate nature of the human disease and presenting welfare implications associated with severe experimental procedures in mammals (ASPA, 1986 amendments 2012). Autologous blood injection and collagenase injection models (Andaluz et al., 2002) are used worldwide, and typical experimental groups include 6-8 rats, sacrificed at various time points for ex vivo histological analysis, which can result in $~ 150$ animals used per publication (Wang et al., 2018) highlighting scope for a change of strategy from a 3 Rs perspective. Unfortunately, this research has not yet resulted in the translation of any specific drugs to the clinic (Kellner \& Connolly, 2010; Kirkman et al., 2011). Potential reasons for this include difficulties in observing cellular responses in 'real-time' within whole brains of intact live animals, and the invasive and artificial procedures required to induce cerebral haematomas (MacLellan et al., 2010; Selim et al., 2018). Mammalian models of spontaneous ICH models do exist, such as cerebral amyloid angiopathy comorbidity studies and use of hypertensive mice, but their usefulness is limited due to variability in haematoma size, timing and location (Alharbi et al., 2016). Alternative and complementary approaches are therefore needed to bridge the "translational gap' for novel drug target discovery in ICH.

Zebrafish (Danio rerio) are becoming an increasingly popular tool for studying cerebrovascular disease (Walcott \& Peterson, 2014). Due to the production of hundreds of offspring from a single adult pairing, zebrafish larvae can be utilised for highthroughput drug screening, thus offering an attractive model for pre-clinical research. Larval transparency and the availability of numerous transgenic reporter lines amount to an extremely powerful system for studying and visualising cellular responses and disease processes in-vivo in real time. Prior to 5 days post fertilisation (dpf), larval zebrafish are not a protected species (in the UK) and could therefore replace a significant number of protected mammals used for pre-clinical study. Furthermore, spontaneous brain-specific bleeding can be observed in zebrafish larvae using non-invasive techniques (Eisa-Beygi et al., 2013; Liu et al., 2007), thereby eliminating specific constraints associated with mammalian models. As such, the use of larval zebrafish models of ICH could offer critical insight into the immediate cellular responses after a bleed to support the rodent community and provide a potential platform for future drug discovery addressing pre-clinical ICH priorities (Selim et al., 2018).

As previously described, zebrafish larvae exposed to atorvastatin (ATV) at 24 hours post-fertilisation (hpf) exhibit spontaneous cerebral-specific blood vessel rupture at the onset of circulation ( $33 \mathrm{hpf}$ ) (Eisa-Beygi et al., 2013; Huang et al., 2018; Li et al., 2017; Shen et al., 2013). Comparably, the 'bubblehead' (bbh) mutant line, which expresses a hypomorphic mutation in the arhgef7 gene, encoding the Rac GEF $\beta$ pix, also exhibit spontaneous ICH and hydrocephalus within a similar time frame to the ATV model (Liu et al., 2007; ten Klooster et al., 2006). ICH is induced through comparable mechanistic defects in both ATV and bbh models (Eisa-Beygi \& Rezaei, 2016). Although several groups have utilised these models to study the development and integrity of the cerebrovasculature 
(Buchner et al., 2007; Huang et al., 2018; Li et al., 2017; Liu et al., 2007; Yang et al., 2017), they have not yet been used to study the pathological and neuroinflammatory consequences of bleeding in the zebrafish larval brain in the context of human ICH. Furthermore, drug intervention studies have focussed on preventing cerebrovascular rupture in zebrafish rather than targeting the disease outcomes, which represent a more realistic therapeutic avenue. In this study, we show that spontaneous ICH in non-protected zebrafish larvae induces quantifiable pathological and inflammatory phenotypes that relate to the human condition. As such, these data support the use of this model species as a valuable complementary system for pre-clinical ICH research.

\section{Methods}

A detailed protocol of the experimental procedure is available in Supplementary File 1

\section{Zebrafish (Danio rerio) strains}

Zebrafish were raised and maintained at The University of Manchester Biological Services Unit under standard conditions as previously described (Westerfield, 2000). Adult zebrafish husbandry was approved by the University of Manchester Animal Welfare and Ethical Review Board. All experiments were performed in accordance with U.K. Home Office regulations (PPL:70/9091) and reported according to ARRIVE guidelines. Transgenic lines used in this study include macrophage-specific lineage mpegl:mCherry (constructed in-house as previously described (Ellett et al., 2011)), neutrophil-specific mpo: GFP (Renshaw et al., 2006), erythroid-specific gatal:dsRed (Traver et al., 2003) and ubiq:secAnnexinV-mVenus, a reporter for apoptosis (re-derived in house (Morsch et al., 2015)) on wild-type, nacre $\left(m i t f a^{\mathrm{w} 2 / \mathrm{w} 2}\right)$ and mutant $\left(b b h^{\mathrm{m} 292}\right)$ backgrounds. Fertilized embryos were collected from natural spawning and incubated at $28^{\circ} \mathrm{C}$ in standard E3 embryo medium and staged according to standard guidelines (Kimmel et al., 1995). At experiment end, zebrafish larvae were terminated prior to protected status using a lethal overdose of MS222 anaesthesia and freezing at $-20^{\circ} \mathrm{C}$.

A completed ARRIVE checklist is available in Supplementary File 2

\section{$\mathrm{ICH}$ models}

ICH was modelled using genetic (bbh) and chemical (ATV) approaches. For the bbh line (Liu et al., 2007), embryos were obtained from adult in-crosses from heterozygous $b b h^{\mathrm{m} 292}$ mutant animals (maintained on wild-type and transgenic reporter backgrounds). For the ATV model, nacre or transgenic embryos were dechorionated at $24 \mathrm{hpf}$ and transferred to clean petri dishes in E3 embryo medium. ATV (Sigma-Aldrich, PZ0001) was solubilised in distilled water to a stock concentration of 0.5 mM. Embryos $(n=100)$ were treated with a final concentration of $1 \mu \mathrm{M}$ ATV through water bath incubation at $28^{\circ} \mathrm{C}$ for 24 hours and equivalent numbers were left as untreated controls. A proportion of ATV-treated embryos did not develop ICH and therefore these animals were used as controls for the treatment (ICH-) alongside untreated (UNT) siblings. For both bbh and ATV models, embryos with evident haemorrhages $(\mathrm{ICH}+)$ were separated from non-haemorrhaged ( $\mathrm{ICH}-$ ) siblings at $\sim 52 \mathrm{hpf}$ for downstream analyses.

\section{Locomotion assay}

Locomotion was measured at $120 \mathrm{hpf}$ to determine if $\mathrm{ICH}$ resulted in a physical phenotype. To remove locomotor function bias, larvae were briefly anesthetised at $72 \mathrm{hpf}$ using $0.02 \%$ MS222 in embryo water and selected at random for plating. Following recovery, $n=24$ larvae were individually transferred to each well of a 24-well plate in $1 \mathrm{ml}$ of fresh methylene-blue-free E3 medium. Cumulative time spent mobile was measured using the DanioVision camera chamber and Ethovision XT software (Noldus, version 11) at room temperature. Analyses were performed on larvae at 72, 96 and $120 \mathrm{hpf}$. Swimming movement of each individual larva was tracked in the $\mathrm{x}$ and $\mathrm{y}$ plane for 10 minutes using a white light stimulus to initiate a startle response every 60 seconds. Cumulative time spent swimming was measured from three independent replicates.

\section{Light sheet microscopy}

Transgenic ICH+ and ICH- larvae were imaged using light sheet microscopy to analyse apoptotic cell death (ubiq:secAnnexinV-mVenus), neutrophils (mpo:GFP) and macrophages (mpeg 1:mCherry). At $\sim 72 \mathrm{hpf}$, randomly selected larvae were anaesthetised using $0.02 \%$ MS222 and mounted in $1.5 \%$ low-melt agarose (Promega), maintained at room temperature. Images were acquired using a W Plan-Apochromat 20X magnification/1.0 UV-VIS objective for light-sheet microscope (Carl Zeiss Lightsheet Z.1) and processed with ZEN imaging software (version 2.3). Maximum intensity projection (MIP) composites were made from $\mathrm{z}$-stack images and brain regions (excluding the eyes) were analysed for average intensity fluorescence of cells with image background removal using an ImageJ (version 1.52a) macro (Supplementary File 1). Numbers of fluorescent cells in the brain were also verified by blind manual counts from MIPs. Data were collected from $n=6-12$ randomly selected larvae per group from 3 independent replicates for ATV studies and verified in two replicates for bbh. For time-lapse recording, MIP composites were stitched from a series of successive z-stack images over a period of 18 hours.

\section{Statistical analysis}

Experimental sample sizes were determined by using power calculations from preliminary data using $\alpha=0.05$ and $\beta=0.80$. All statistical analysis was first performed using GraphPad Prism 7.0 and then verified using R (R Core Team, 2018) for non-parametric data and subsequent significance values plotted. Linear mixed modelling (LMM) was used to evaluate the effects of independent factors on the continuous dependent variables (Bates et al., 2015). All factors and interactions were modelled as fixed effects. As there is a lack of independence in fish from the same clutch, "Clutch" was treated as a random effect, modelled with random intercepts for all models. The significance of inclusion of an independent variable or interaction terms were evaluated using log-likelihood ratio. Holm-Sidak post-hocs were then performed for pair-wise comparisons using the least square means (Lenth, 2016). Homoscedasticity and normality of the Pearson residuals were evaluated graphically using predicted vs residual and Q-Q plots, respectively, and transformations were applied when necessary. 
For discrete data and data with non-normal distributions, generalized linear mixed modelling was used (GLMM) (Bates et al., 2015; Fournier et al., 2012; Skaug et al., 2013). Again "Clutch" was treated as a random effect modelled with random intercepts for all models. Appropriate families were selected based on the data distribution. Numerous families and link functions were evaluated where necessary and the optimal parameters were selected based on the Akaike information criterion (AIC). For mobile or non-mobile (yes/no) data, a logistic regression with cloglog link was selected; for count data (number of dead cells) a negative binomial family was selected. The significance of inclusion of an independent variable or interaction terms were evaluated using log-likelihood ratio. Holm-Sidak post-hocs were then performed for pair-wise comparisons using the least square means (Lenth, 2016). Pearson residuals were evaluated graphically using predicted vs level plots. All analyses were performed using R ( $\mathrm{R}$ Core Team, 2018) (Supplementary File 3).

\section{Results}

ATV and bbh mutant models show comparable cerebral bleeding phenotypes

It has been shown that both the ATV and bbh models share similar underlying mechanisms that are responsible for neuroendothelial weakness in the developing larvae and spontaneous cranial vessel rupture (Eisa-Beygi et al., 2013; Liu et al., 2012). In this study we compared these models in the context of ICH and characterised the pathological outcomes of haemorrhage to develop a new platform for pre-clinical interrogation of postbleed consequences. Using the translucent nature of the zebrafish embryo we observed brain-specific bleeding non-invasively, using light and fluorescent microscopy (Figure 1A). Bleeds were observed in fore, mid and hindbrain regions at comparable frequencies, as described by others (Eisa-Beygi et al., 2013; Liu et al., 2007). ATV absorption induced haemorrhages in a dose-dependent manner when embryos were treated at 24 hpf (Figure 1B). Homozygous mutant bbh embryos and ATV-treated embryos both exhibited ICH between 38 and $48 \mathrm{hpf}$ (Figure 1C, D). In homozygous mutant bbh embryos, ICH was frequently accompanied with severe cranial oedema (Liu et al., 2007) (Supplementary Figure 1). Wild-type and heterozygous bbh siblings had no haemorrhages and were utilised as ICH-controls.

$\mathrm{ICH}$ in zebrafish larvae results in a quantifiable brain injury To determine the pathological consequences of ICH in zebrafish larvae, we next assessed brain injury using a transgenic ubiq: secAnnexinV-mVenus cell death reporter line (Morsch et al., 2015). AnnexinV binding was assessed between 48 and 120 hpf, to characterise the timeline of cell death following an $\mathrm{ICH}$ event (Supplementary Figure 2). We observed peri-haematomal brain-damaged lesions as 'clusters' of dying cells formed by $72 \mathrm{hpf}$, which had receded before $96 \mathrm{hpf}$ (Supplementary Figure 2). We quantified brain lesions from images taken at 72 hpf (Figure 2A). In both ATV and bbh models, bleeding was associated with a significant, two-fold increase in intensity of fluorescent signal in the brain compared to ICH- controls (Figure 2B, C). These data were verified using blinded, manual counts of annexinV-positive cells from MIP images (Supplementary Figure 3). Taken together, these data provide convincing evidence that ICH in zebrafish larvae induces a reproducible cell death phenotype that can act as a quantifiable readout of brain injury.

$\mathrm{ICH}$-induced brain injury results in a quantifiable locomotor deficit in zebrafish larvae

To investigate whether ICH-induced brain injury was associated with a locomotor deficit, as frequently seen in stroke patients (Kloter et al., 2011; Saulle \& Schambra, 2016), we tracked swimming behaviour between 72 and 120 hpf. Larvae were analysed across 3 days to account for the improvement in baseline swimming performance associated with increasing age. Representative tracks are shown in Figure 3A. ICH+ larvae spent significantly less time swimming in the cumulative time spent mobile during the 10 minute recording period at both 72 and 96 hpf compared to ICH- siblings, implying a persistent physical deficit (Figure 3B). Although a reduction was observed in ICH+ larvae at $120 \mathrm{hpf}$, this was not significantly different to ICH- siblings $(\mathrm{p}=0.08)$. However ATV-treated larvae assayed at $120 \mathrm{hpf}$ did show a significant reduction in swimming in $\mathrm{ICH}+$ larvae and not in controls. Comparable motility phenotypes in both models imply that the swimming deficit is due to cerebral bleed and not statin treatment. Reproducing these results in both models suggests that the impairment in locomotion is not caused by any one mechanistic factor but due to ICH itself.

\section{$\mathrm{ICH}$-induced brain injury in zebrafish larvae initiates an innate immune response}

In order to determine whether ICH in zebrafish larvae initiated inflammation at the cellular level, neutrophils and macrophages in the brain were quantified using the mpo:GFP and mpeg1: mCherry reporter lines. At $72 \mathrm{hpf}$, the number of macrophages increased significantly, doubling in the brains of $\mathrm{ICH}+$ larvae compared to ICH- siblings (Figure 4). At the same time point, neutrophil numbers were greater in $\mathrm{ICH}+$ larvae than $\mathrm{ICH}$ - larvae; however, the difference did not reach significance.

\section{Activated macrophage cells show a phagocytic response} to the brain lesion

We investigated the phagocytic response of activated macrophages to the brain lesion sites in ubiq:secAnnexinV-mVenus; $m p e g 1$ : mCherry $\mathrm{ICH}+$ larvae using real time microscopy. The formation of a new brain lesion site was recorded between 55 and 65 hpf (Supplementary Video 1) and macrophages were seen migrating to sites of injury and actively phagocytosing annexinV positive dying cells (Figure 5A). We next analysed total macrophage activity within the brain, using morphology as an indication of phagocytic activation. Amoeboid, rounded cells were considered phagocytic and ramified cells considered inactive, as previously defined (Morsch et al., 2015). We found a substantial increase in the proportion of activated, amoeboid macrophages in the $\mathrm{ICH}+$ brain compared to $\mathrm{ICH}$ - siblings (Figure 5B, C). These results indicate that the innate immune response to $\mathrm{ICH}$ can be examined at the cellular level in zebrafish ICH models using real-time microscopy. 
A

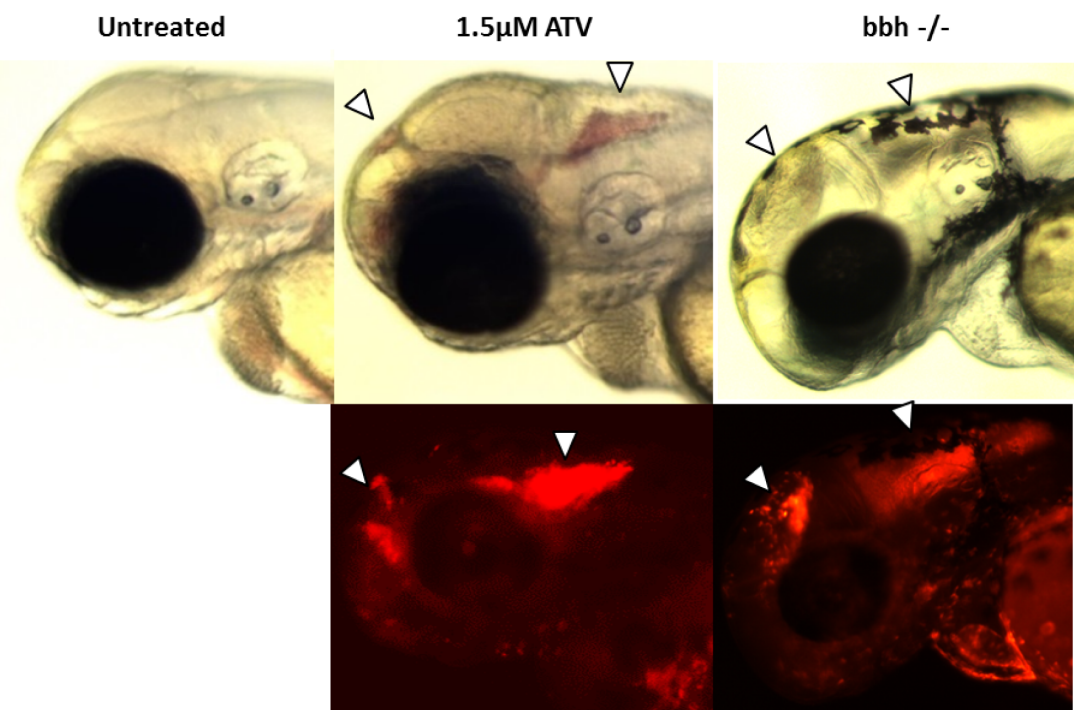

B

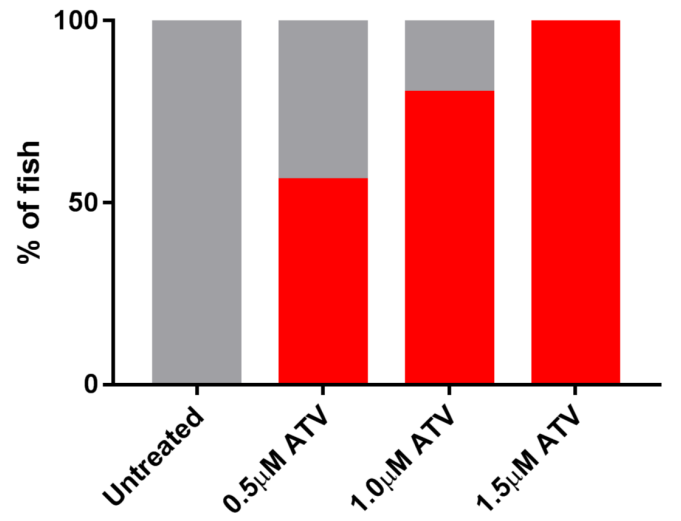

$\mathrm{ICH}-$

bbh

D

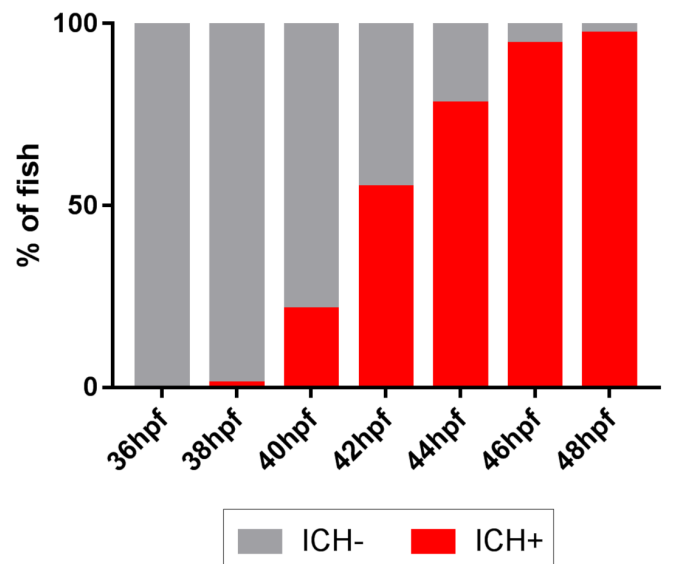

C

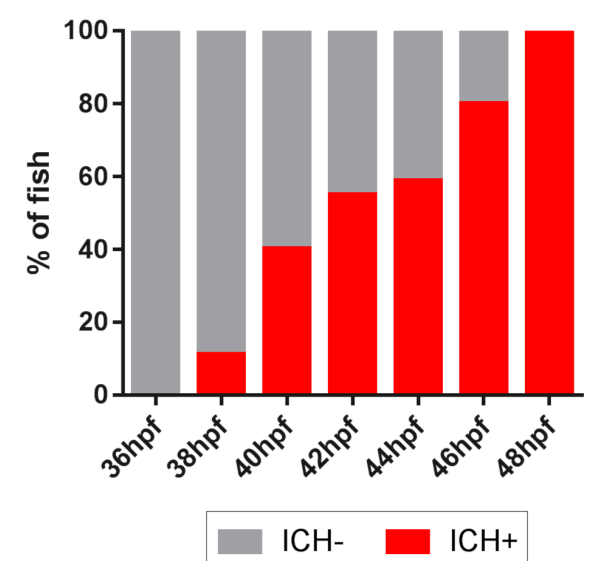

Figure 1. Atorvastatin (ATV)-induced and bubblehead (bbh) mutant intracerebral haemorrhage (ICH) show comparable models of brain-specific bleeding. (A) Brain-specific bleeds were observed in both ATV and bbh models maintained on the transgenic gata1:DsRed reporter background using both brightfield (top panels) and fluorescence (bottom panels) microscopy. Bleeds formed in both forebrain and mid-hindbrain regions, as described by others (Eisa-Beygi et al., 2013) (arrows denotes haemorrhages). Bleeds in bbh mutants are frequently associated with severe cranial oedema making blood pooling more disperse. Original magnification, x20. (B) ATV treatment causes ICH to occur in a dose-dependent manner. (C) Timeline of ICH development in ATV-treated and untreated embryos and (D) bbh homozygotes. 
A
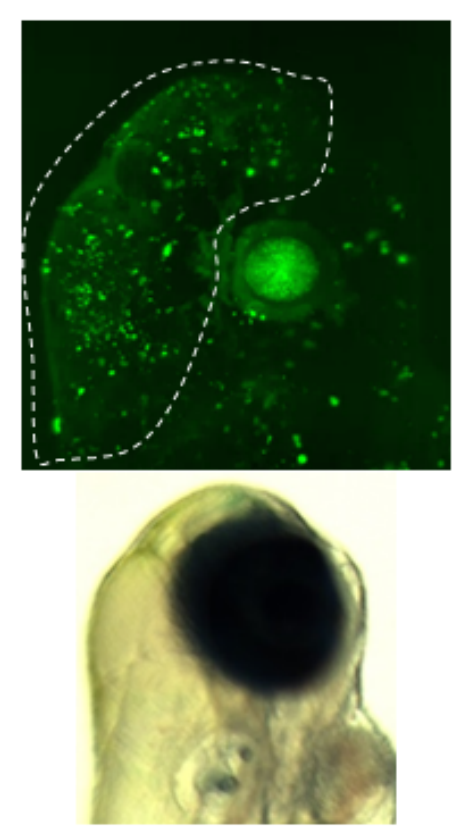

B

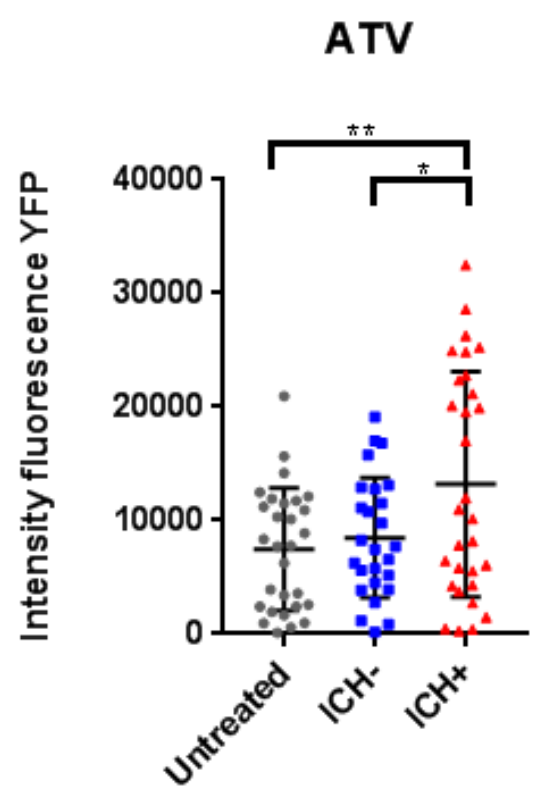

$\mathrm{ICH+}$
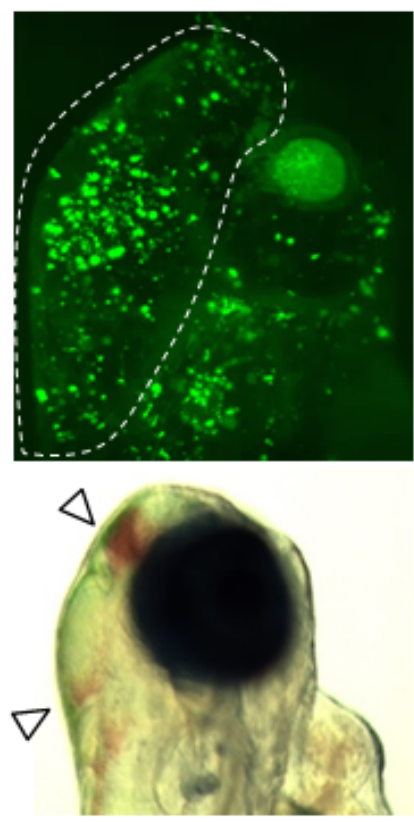

C bbh

Figure 2. Intracerebral haemorrhage (ICH) in zebrafish larvae results in a quantifiable brain injury. (A) Representative images of the brain injury phenotype in $\mathrm{ICH}+$ larvae (right panels), in comparison to $\mathrm{ICH}$ - siblings (left panels), at 72 hpf. Bright-field images (bottom panels) demonstrate the presence of brain bleeds (arrows) in $\mathrm{ICH}+$ larvae. Fluorescent microscopy was performed to visualise cell death in the ubiq: secAnnexinV-mVenus reporter line (top panels). Clusters of dying cells were observed in peri-haematomal regions. Images were cropped to brain only regions and analysed for total green fluorescence intensity in round particles bigger than 30 pixels in diameter (white line). (B) Quantification of fluorescent signal in the brains of untreated, $\mathrm{ICH}$ - and $\mathrm{ICH}+$ larvae obtained through the ATV model ( $\mathrm{n}=12$ per group; 3 independent replicates) at $72 \mathrm{hpf}$. Significant differences were observed when comparing $\mathrm{ICH}+$ with untreated (** $\mathrm{p}=0.004)$ and with $\mathrm{ICH}-\left({ }^{*} \mathrm{p}=0.03\right)$ siblings. (C) Quantification of fluorescent signal as a read out for annexinV binding in the brains of $\mathrm{ICH}$ - and $\mathrm{ICH}+$ larvae obtained through the bubblehead (bbh) model ( $\mathrm{n}=12$ per group; 2 independent replicates) at 72 hpf. A significant difference in mVenus fluorescence was observed between $\mathrm{ICH}+$ and $\mathrm{ICH}$ - age-matched siblings ( ${ }^{\star \star} \mathrm{p}=0.002$ ). Original magnification, $\mathrm{x} 20$. 


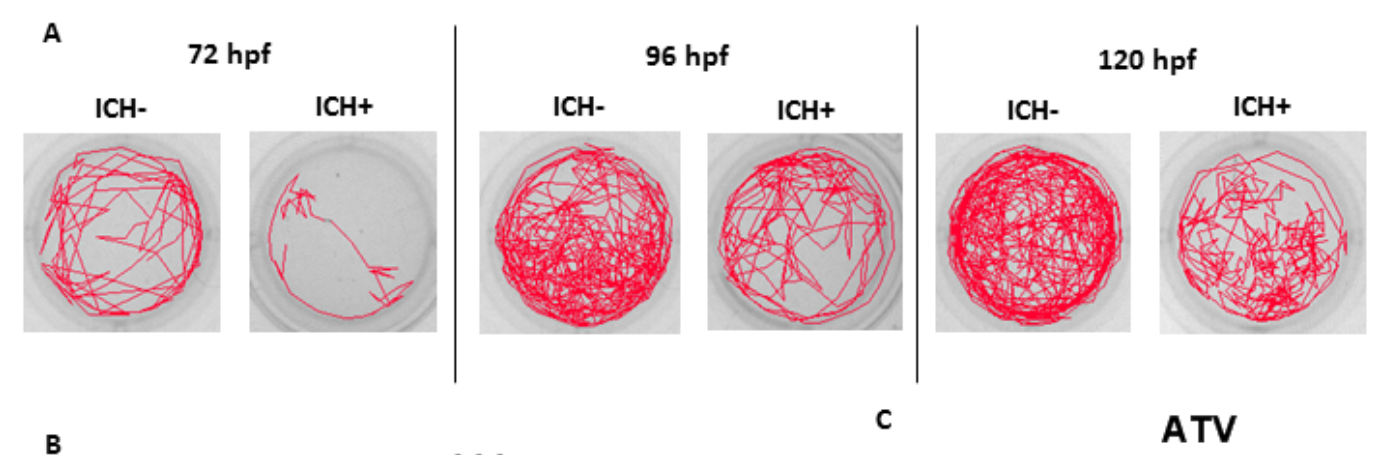

B

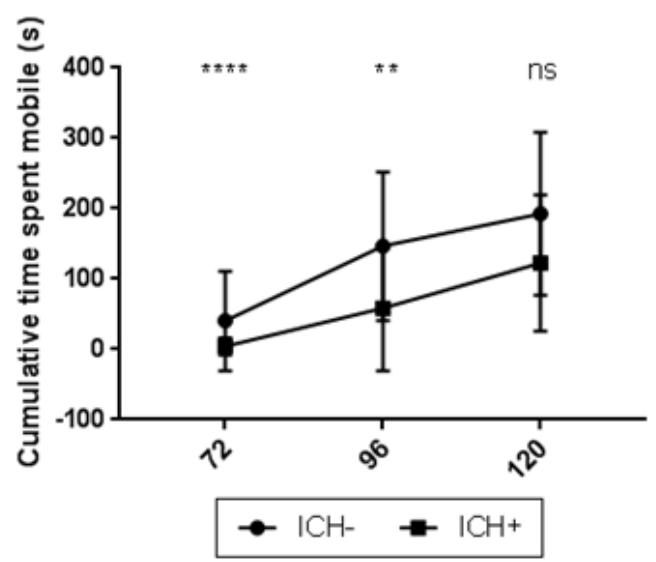

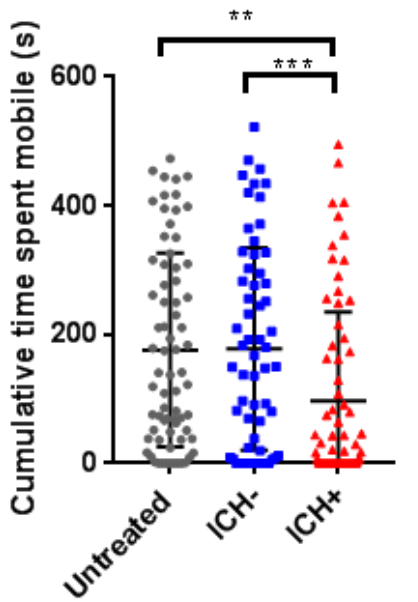

Figure 3. ICH-induced brain injury results in a quantifiable locomotor deficit in bubblehead (bbh) zebrafish larvae. (A) Representative examples of the swimming tracks in $\mathrm{ICH}$ - and $\mathrm{ICH}+$ larvae at 72, 96 and $120 \mathrm{hpf}$. (B) $\mathrm{ICH}+$ larvae exhibited a significant decrease in the cumulative time spent mobile during the 10 minute recording period at both 72 and 96 hpf. Significance was lost at the 120 hpf time point potentially alluding to recovery from brain injury ( $n=24$ larvae per group; 3 independent replicates; ${ }^{\star \star \star \star} p=0.00006 ;{ }^{* *} p=0.003 n s p=0.08$ ) (C) Quantification of cumulative time spent moving in untreated and ATV-treated ICH- and ICH+ larvae at $120 \mathrm{hpf}$. ICH+ larvae exhibited a significant decrease in the cumulative time spent mobile during the 10 minute recording period. Three technical replicates ( $n=24$ larvae per group) were used to calculate s.d from the mean $\left({ }^{* *} \mathrm{p}=0.00004,{ }^{* \star} \mathrm{p}=0.0003\right)$.

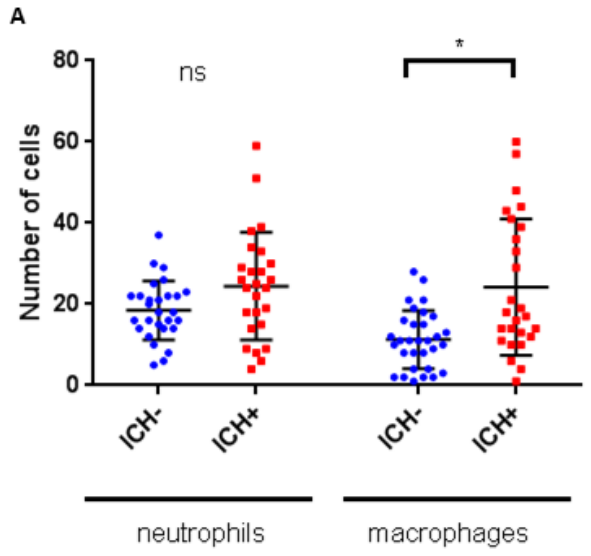

Figure 4. Intracerebral haemorrhage (ICH) initiates an innate cellular immune response in the zebrafish larval brain. Numbers of leukocytes quantified within the brains of mpo:GFP;mpeg1: dsRed double transgenic larvae ( $n=8$ per group; 2 independent replicates) at $72 \mathrm{hpf}$ reveals a significant increase in macrophages $\left({ }^{*} p=0.01\right)$, but not neutrophils $(p=0.5)$, in response to $\mathrm{ICH}$.

\section{Dataset 1. All raw data from the present study}

https://dx.doi.org/10.5256/f1000research.16473.d220415

Data include all raw microscopy images of cell death in Annexin and bubblehead groups; fluorescence intensities, cell counts and motility times for ATV and bubblehead groups; and leukocyte cell counts.

\section{Discussion}

Here, we reveal that ICH in zebrafish larvae induces quantifiable pathological and inflammatory consequences that mimic aspects of human pathophysiology. Several groups have previously described the mechanisms underlying neurovascular instability in zebrafish larval models of ICH (Buchner et al., 2007; Eisa-Beygi et al., 2013; Liu et al., 2007), and drug intervention studies have attempted to identify compounds that can inhibit cerebral bleeding (Li et al., 2017; Yang et al., 2017). However, the relevance and translational impact of intervention before onset of haemorrhage is unclear. As such, we focussed our attention on characterising the pathological and immunological 
A
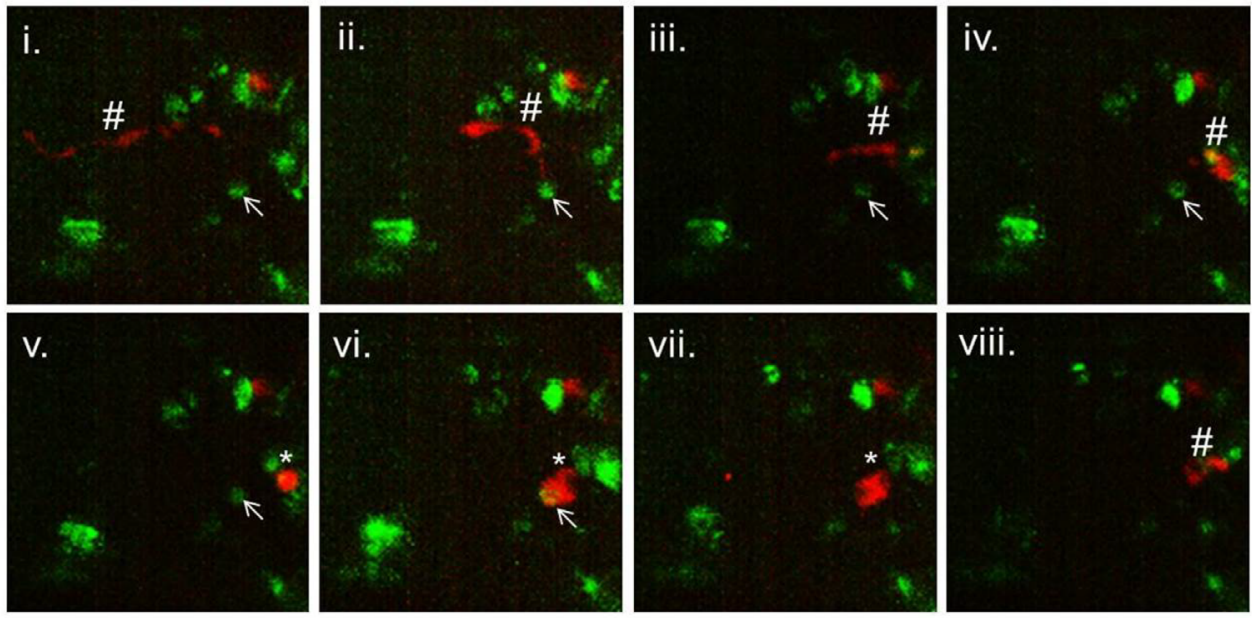

B

$\mathrm{ICH}-$

$\mathrm{ICH+}$
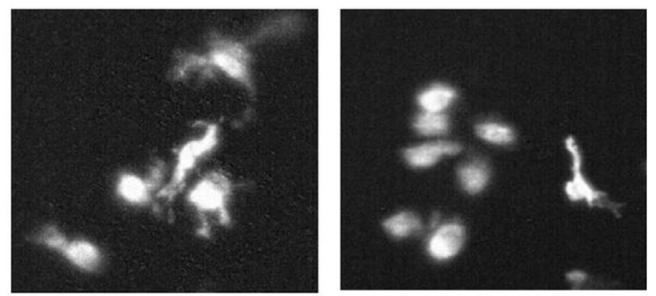

C

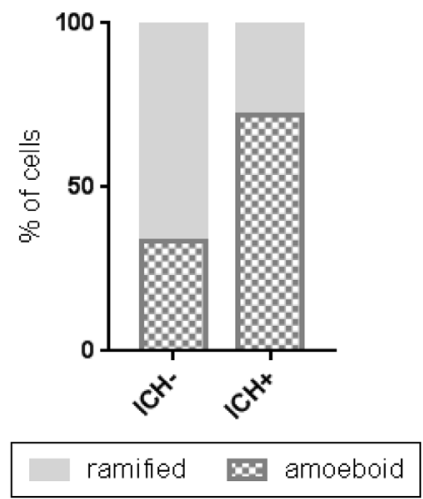

Figure 5. Activated macrophage cells show a phagocytic response to the brain lesion. (A) Representative time-lapse stills (from Supplementary Video 1) showing a ramified patrolling macrophage migrating towards an annexinV positive cell ( $i$ - vi). The macrophage acquired an amoeboid morphology (v) before phagocytosing the annexinV-positive cell (vi, vii). After phagocytosis the macrophage resumes a ramified morphology and migrates away and the annexinV-positive cell can no longer be seen (viii). Ramified macrophage (\#), annexinV positive cell (arrow), amoeboid macrophage $\left(^{*}\right)$. (B) Representative images of mpeg1-positive cells in the intracerebral haemorrhage (ICH)and $\mathrm{ICH}+$ larval brain exhibiting amoeboid and ramified morphologies. (C) An increased proportion of amoeboid (phagocytic) and decreased proportion of ramified (inactive) macrophages was observed in $\mathrm{ICH}+$ brains in comparison to $\mathrm{ICH}$ - siblings.

consequences of blood in the brain in zebrafish models, which we consider to be a more realistic therapeutic target.

In general, ICH can be predominantly considered as a disorder associated with older age. Consequently, it is remarkable that the phenotypes observed in developing zebrafish recapitulate those associated with the aged human brain, indicating the innate injury response to blood in the brain is both evolutionarily conserved between species and analogous during development and adulthood. Zebrafish models have been employed in other neurological and neuropsychiatric disease investigation, including epilepsy, schizophrenia, Alzheimer's and Parkinson's disease, because of these conserved fundamental mechanisms and behaviours (Fontana et al., 2018; Vaz et al., 2018). Although these models present their own limitations, the use of noninvasive in vivo imaging, ease of genetic manipulation and availability of large sample sizes offers new insights and overcomes some of the common restrictions associated with rodent 
models. Given that ICH occurs spontaneously using non-invasive techniques in zebrafish, it can be argued this system more accurately models some aspects of the human condition than the most commonly used rodent models (Andaluz et al., 2002; Rosenberg et al., 1990). The use of rodents has not been successful in terms of identifying translatable therapeutic targets for ICH. As such, we propose that the post-ICH pathologies presented in this study (Figure 6) represent an alternative, complementary platform for pre-clinical ICH research and future drug discovery.

The transferability of employing these zebrafish larval models would address the 3Rs welfare issue of prolonged severe surgical procedures in mammals by replacing some of these animals with zebrafish larvae of unprotected status. We have used equipment and procedures that are commonplace and would be relatively simple to adopt in other labs. We would like to see these models used to determine the translatability of drugs that prevent cerebral bleeding in zebrafish (Huang et al., 2018; Yang et al., 2017), to investigate the clinical relevance of post-ICH treatment. We also propose these endpoint assays can be used to develop medium/high-throughput drug screening to identify new compounds for pre-clinical investigation.

In humans, an influx of blood into the brain causes primary brain injury through neuronal death and cell necrosis, inducing a secondary phase of injury triggered by the production of inflammatory mediators and innate immune cell migration towards the site of injury (Mracsko \& Veltkamp, 2014). We show that cerebral bleeding in zebrafish larvae causes an increase in cell death in the brain not shown before in zebrafish haemorrhage models. This lesion was associated with a physical impairment, as observed by a reduction in swimming ability, which we propose is either due to a defect in stimulus perception or through a motor deficiency. This impairment is seen to begin to recover at 3 days post injury, suggesting the zebrafish larval model system as a useful tool for further investigation to reveal recovery processes responding to brain cell death. Importantly, these observations mimic outcomes that are exhibited in rodent models (MacLellan et al., 2008) and ICH patients (Saulle \& Schambra, 2016). In clinical presentation of ICH the initial haematoma mass effect and increase in intracranial pressure exacerbates brain damage, compressing surrounding structures (Xi et al., 2006) and increases risk of death following ICH (Yang et al., 2015). In zebrafish larvae, cerebral oedema is regularly associated with ICH (Kasher et al., 2015; Liu et al., 2007). However without a fully developed cranium, oedema is unlikely to result in the same injury severity seen in humans, which may be one limitation of this particular model system.

An increase in recruitment and activation of macrophages in the brain was also observed in zebrafish following haemorrhage corresponding to time of cell death. A trend towards increased recruitment of neutrophils was also observed, but this result did not reach statistical significance. It is possible that a rapid temporal neutrophil response was not fully captured during our analytical time frame. However, it has been shown that neutrophils are not involved in the clearance of cellular debris in a zebrafish larval model of brain injury (van Ham et al., 2014), suggesting neutrophils are less vital to early injury responses in the brain than macrophages. It remains unclear whether activation of macrophages post-ICH is overall beneficial or deleterious in the short term after injury. Pro-inflammatory cells contribute to the breakdown of the blood-brain barrier (Abbott, 2000); however, phagocytic cells promote clearance of red blood cells and tissue debris, which occurs from 7 days post haemorrhage in rodent models of ICH (Mracsko \& Veltkamp, 2014; Yang et al., 2016b). Early responses to

Eggs harvested

bbh

\begin{tabular}{|c|c|c|c|c|c|}
\hline Ohpf & $24 \mathrm{hpf}$ & 48hpf & 72hpf & 96hpf & 120hpf \\
\hline \multirow[t]{2}{*}{$\begin{array}{l}\text { Eggs from bbh } \\
\text { +/- pairing } \\
\text { harvested }\end{array}$} & & $\begin{array}{l}100 \% \text { bbh }-/- \\
\text { ICH+ with } \\
\text { severe oedema }\end{array}$ & $\begin{array}{c}\text { Annexin +ve } \\
\text { cell clusters } \\
\text { observable }\end{array}$ & & $\begin{array}{c}\text { Locomotior } \\
\text { assay }\end{array}$ \\
\hline & & $\begin{array}{l}\text { ICH- siblings } \\
\text { separated }\end{array}$ & $\begin{array}{c}\text { Cellular } \\
\text { neuroinflamm- } \\
\text { atory reaction }\end{array}$ & & $\begin{array}{l}\text { Larvae } \\
\text { terminated }\end{array}$ \\
\hline
\end{tabular}

Figure 6. Graphic of experimental timeline to characterise brain injury, locomotor and neuroinflammatory outcomes. ICH, intracerebral haemorrhage; bbh, bubblehead. 
laser-induced cerebral bleeding in zebrafish have shown that macrophages are essential for vessel repair (Liu et al., 2016) and in zebrafish stab wound brain injury models, inflammation is necessary for regeneration and recovery (Kyritsis et al., 2012). Studies show that polarisation of macrophages to M1-like and M2-like states change over time in rodent models of ICH in response to brain damage molecules (Wan et al., 2016; Yang et al., 2016a) and some drugs in clinical trials target microglial polarisation in an attempt to prevent the pro-inflammatory phenotype (Lan et al., 2017). In vivo imaging of ICH-induced inflammatory processes has barely been explored (Mracsko \& Veltkamp, 2014), and so the translucent nature of zebrafish larvae and availability of transgenic lines offer an accessible model for further interrogation. Observations of cellular interactions within whole, intact rodent brains at ongoing time points are currently not possible, therefore utilising the zebrafish system will allow us to learn more about leukocyte behaviour after spontaneous ICH. Furthermore, immune responses observed in real time in this system will have been elicited by spontaneous vessel rupture and not as an artefact of $\mathrm{ICH}$ surgery or ex vivo analysis (Kirkman et al., 2011; Xue \& Del Bigio, 2003). For better translation of therapies from pre-clinical to patients, understanding of early innate immune responses to spontaneous bleeding needs to be improved, thus zebrafish offer a powerful resource to facilitate this.

In conclusion, given the advantages associated with zebrafish larvae and the potential for a $3 \mathrm{Rs}$ approach to pre-clinical stroke research, we propose that this model organism can provide critical insight into ICH pathophysiology during the early phases of injury and offers a future platform for drug discovery.

\section{Data availability}

Dataset 1. All raw data from the present study. Data include all raw microscopy images of cell death in Annexin and bubblehead groups; fluorescence intensities, cell counts and motility times for ATV and bubblehead groups; and leukocyte cell counts. DOI: https://doi.org/10.5256/f1000research.16473. d220415 (Crilly et al., 2018).

\section{Grant information}

This study was supported by the NC3Rs (NC/N002598/1), Stroke Association (TSA LECT 2017/02), ERA-NET NEURON (MR/M501803/1) and The British Heart Foundation (FS/14/64/31291, FS/15/67/32038). We are also particularly thankful to The Natalie Kate Moss Trust for their continued financial support

The funders had no role in study design, data collection and analysis, decision to publish, or preparation of the manuscript.

\section{Acknowledgements}

We would like to thank Prof Yanick Crow and Dr Gillian Rice for use of equipment and reagents, Dr David Spiller and the University of Manchester Systems Microscopy Core Facility for use of the light sheet microscope, and Prof Richard Baines for the use of DanioVision. The bbh line was kindly shared by Nicole Munsie from Dr Sarah Child's lab at the University of Calgary. We also thank Prof. Stephen Renshaw for sharing the mpo:GFP line.

\section{Supplementary material Supplementary File 1. Full detail protocol for experimental methodology. \\ Click here to access the data}

Supplementary File 2. Completed ARRIVE guidelines checklist.

Click here to access the data

\section{Supplementary File 3. Supplementary statistical analysis.}

Click here to access the data

Supplementary Figure 1. Severe cranial oedema (affected regions denoted by hashtags) associated with cranial bleeds in 'bubblehead' homozygous mutants (right panel) and subsequently exhibit more dispersed bleeds (arrows) compared to heterozygous and wild-type intracerebral haemorrhage (ICH) (left panel).

Original magnification, $\mathrm{x} 20$.

Click here to access the data

Supplementary Figure 2. Representative images of the brain injury phenotype in intracerebral haemorrhage (ICH)+ larvae (bottom panels), in comparison to ICH- age-matched siblings (top panels), at 48-120 hpf.

Fluorescent microscopy was performed to visualise cell death in the ubiq:secAnnexinV-mVenus reporter line to determine the timeline of cell injury following an ICH event. Original magnification, x20.

Click here to access the data 
Supplementary Figure 3. Manual counts of fluorescent cells from blinded analysis of the ubiq:secAnnexinV-mVenus reporter line in atorvastatin (ATV) and bubblehead (bbh) models show a significant increase in intracerebral haemorrhage $(\mathrm{ICH})+(* * \mathrm{p}=0.0097$, $\# \#$ \#=0.0005).

Click here to access the data

Supplementary Video 1. A time lapse recording of phagocytosis occurring in an intracerebral haemorrhage+ ubiq:secAnnexinVmVenus;mpeg1:mCherry larvae between 55 and 72 hpf.

Cluster of dying cells (green annexinV positive) in forebrain visible at $55 \mathrm{hpf}$ and active macrophages (red) can be observed phagocytosing cells. A second cluster in the mid brain develops over recording period and macrophages can be seen migrating to the new lesion site. Original magnification, $\mathrm{x} 20$.

Click here to access the data

Abbott $\mathrm{NJ}$ : Inflammatory mediators and modulation of blood-brain barrier permeability. Cell Mol Neurobiol. 2000; 20(2): 131-147. PubMed Abstract

Alharbi BM, Tso MK, Macdonald RL: Animal models of spontaneous intracerebral hemorrhage. Neurol Res. 2016; 38(5): 448-455. PubMed Abstract | Publisher Full Text

An SJ, Kim TJ, Yoon BW: Epidemiology, Risk Factors, and Clinical Features of Intracerebral Hemorrhage: An Update. J Stroke. 2017; 19(1): 3-10.

PubMed Abstract | Publisher Full Text | Free Full Text

Andaluz N, Zuccarello M, Wagner KR: Experimental animal models of intracerebral hemorrhage. Neurosurg Clin N Am. 2002; 13(3): 385-393. PubMed Abstract | Publisher Full Text

ASPA 1986 amendments 2012: Animals (Scientific Procedures) Act 1986. Reference Source

Bates D, Mächler M, Bolker B, et al.: Fitting Linear Mixed-Effects Models using Ime4. J Stat Softw. Computation. 2015; 67(1): 1-48.

Publisher Full Text

Buchner DA, Su F, Yamaoka JS, et al.: pak2a mutations cause cerebral hemorrhage in redhead zebrafish. Proc Natl Acad Sci U S A. 2007; 104(35): 13996-14001.

PubMed Abstract | Publisher Full Text | Free Full Text

Casals JB, Pieri NC, Feitosa ML, et al.: The use of animal models for stroke research: a review. Comp Med. 2011; 61(4): 305-313.

PubMed Abstract | Free Full Text

Crilly S, Njegic A, Laurie SE, et al: Dataset 1 in: Using zebrafish larval models to study brain injury, locomotor and neuroinflammatory outcomes following intracerebral haemorrhage. F1000Research. 2018.

http://www.doi.org/10.5256/f1000research.16473.d220415

Eisa-Beygi S, Hatch G, Noble S, et al:: The 3-hydroxy-3-methylglutaryl-CoA reductase (HMGCR) pathway regulates developmental cerebral-vascular stability via prenylation-dependent signalling pathway. Dev Biol. 2013; 373(2): 258-266.

PubMed Abstract | Publisher Full Text

Eisa-Beygi S, Rezaei M: Etiology of intracerebral hemorrhage (ICH): nove insights from Zebrafish embryos. Int J Dev Biol. 2016; 60(4-6): 119-126. PubMed Abstract | Publisher Full Text

Ellett F, Pase L, Hayman JW, et al: mpeg1 promoter transgenes direct macrophage-lineage expression in zebrafish. Blood. 2011; 117(4): e49-e56. PubMed Abstract | Publisher Full Text | Free Full Text

Fontana BD, Mezzomo NJ, Kalueff AV, et al.: The developing utility of zebrafish models of neurological and neuropsychiatric disorders: A critical review. Exp Neurol. 2018; 299(Pt A): 157-171.

PubMed Abstract | Publisher Full Text

Fournier DA, Skaug HJ, Ancheta J, et al:: AD Model Builder: using automatic differentiation for statistical inference of highly parameterized complex nonlinear models. Optim Methods Softw. 2012; 27(2): 233-249.

Publisher Full Text

Huang B, Zhou ZY, Li S, et al.: Tanshinone I prevents atorvastatin-induced cerebral hemorrhage in zebrafish and stabilizes endothelial cell-cell adhesion by inhibiting VE-cadherin internalization and actin-myosin contractility.

Pharmacol Res. 2018; 128: 389-398.

PubMed Abstract | Publisher Full Text

Kasher PR, Jenkinson EM, Briolat V, et al: Characterization of samhd1 morphant zebrafish recapitulates features of the human type I interferonopathy AicardiGoutières syndrome. J Immunol. 2015; 194(6): 2819-2825.

PubMed Abstract | Publisher Full Text
Kellner CP, Connolly ES Jr: Neuroprotective strategies for intracerebral hemorrhage: trials and translation. Stroke. 2010; 41(10 Suppl): S99-S102. PubMed Abstract | Publisher Full Text

Kimmel CB, Ballard WW, Kimmel SR, et al:: Stages of embryonic development of the zebrafish. Dev Dyn. 1995; 203(3): 253-310.

PubMed Abstract | Publisher Full Text

Kirkman MA, Allan SM, Parry-Jones AR: Experimental intracerebral hemorrhage: avoiding pitfalls in translational research. J Cereb Blood Flow Metab. 2011; 31(11): 2135-2151.

PubMed Abstract | Publisher Full Text | Free Full Text

Kloter E, Wirz M, Dietz V: Locomotion in stroke subjects: interactions between unaffected and affected sides. Brain. 2011; 134(Pt 3): 721-731.

PubMed Abstract | Publisher Full Text

Kyritsis N, Kizil C, Zocher S, et al:: Acute inflammation initiates the regenerative response in the adult zebrafish brain. Science. 2012; 338(6112): 1353-1356. PubMed Abstract | Publisher Full Text

Lan X, Han X, Li Q, et al:: Modulators of microglial activation and polarization after intracerebral haemorrhage. Nat Rev Neurol. 2017; 13(7): 420-433.

PubMed Abstract | Publisher Full Text | Free Full Text

Lenth RV: Least-squares means: the R package Ismeans. J Stat Softw. 2016; 69(1): 1-33.

Publisher Full Text

Li S, Ai N, Shen M, et al.: Discovery of a ROCK inhibitor, FPND, which prevents cerebral hemorrhage through maintaining vascular integrity by interference with VE-cadherin. Cell Death Discov. 2017; 3: 17051.

PubMed Abstract | Publisher Full Text | Free Full Text

Liu J, Fraser SD, Faloon PW, et al:: A betaPix Pak2a signaling pathway regulates cerebral vascular stability in zebrafish. Proc Natl Acad Sci U S A. 2007; 104(35): 13990-13995.

PubMed Abstract | Publisher Full Text | Free Full Text

Liu C, Wu C, Yang Q, et al:: Macrophages Mediate the Repair of Brain Vascular Rupture through Direct Physical Adhesion and Mechanical Traction. Immunity. 2016; 44(5): 1162-1176.

PubMed Abstract | Publisher Full Text

Liu J, Zeng L, Kennedy RM, et al.: $\beta$ Pix plays a dual role in cerebral vascular stability and angiogenesis, and interacts with integrin $\alpha_{v} \beta_{8}$. Dev Biol. 2012; 363(1): 95-105

PubMed Abstract | Publisher Full Text

Lok J, Leung W, Murphy S, et al:: Intracranial hemorrhage: mechanisms of secondary brain injury. Acta Neurochir Suppl. Intracerebral Hemorrhage Research. Springer. 2011; 111: 63-9.

PubMed Abstract | Publisher Full Text | Free Full Text

MacLellan CL, Silasi G, Auriat AM, et al.: Rodent models of intracerebral hemorrhage. Stroke. 2010; 41(10 Suppl): S95-S98.

PubMed Abstract | Publisher Full Text

MacLellan CL, Silasi G, Poon CC, et al.: Intracerebral hemorrhage models in rat: comparing collagenase to blood infusion. J Cereb Blood Flow Metab. 2008; 28(3): 516-525.

PubMed Abstract | Publisher Full Text

Morsch M, Radford R, Lee A, et al:: In vivo characterization of microglial engulfment of dying neurons in the zebrafish spinal cord. Front Cell Neurosci. 2015; 9: 321. PubMed Abstract | Publisher Full Text | Free Full Text

Mracsko E, Veltkamp R: Neuroinflammation after intracerebral hemorrhage. Front Cell Neurosci. 2014; 8: 388.

PubMed Abstract | Publisher Full Text | Free Full Text

Renshaw SA, Loynes CA, Trushell DM, et al:: A transgenic zebrafish model of 
neutrophilic inflammation. Blood. 2006; 108(13): 3976-3978. PubMed Abstract | Publisher Full Text

Rosenberg GA, Mun-Bryce S, Wesley M, et al.: Collagenase-induced intracerebral hemorrhage in rats. Stroke. 1990; 21(5): 801-807.

PubMed Abstract | Publisher Full Text

Saulle MF, Schambra HM: Recovery and Rehabilitation after Intracerebral

Hemorrhage. Semin Neurol. NIH Public Access, 2016; 36(3): 306-12.

PubMed Abstract | Publisher Full Text | Free Full Text

Selim M, Hanley D, Broderick J, et al.: Basic and Translational Research in Intracerebral Hemorrhage: Limitations, Priorities, and Recommendations. Stroke. 2018; 49(5): 1308-1314.

PubMed Abstract | Publisher Full Text | Free Full Text

Shen M, Yu H, Li Y, et al.: Discovery of Rho-kinase inhibitors by docking-based virtual screening. Mol Biosyst. 2013; 9(6): 1511-1521.

PubMed Abstract | Publisher Full Text

Skaug $\mathrm{H}$, Fournier D, Nielsen A, et al.: Generalized linear mixed models using AD model builder. $R$ package version 0.7. 2013; 7 .

Team RC: R: A Language and Environment for Statistical Computing. 2018. Reference Source

ten Klooster JP, Jaffer ZM, Chernoff J, et al:: Targeting and activation of Rac1 are mediated by the exchange factor beta-Pix. J Cell Biol. 2006; 172(5): 759-769.

PubMed Abstract | Publisher Full Text | Free Full Text

Traver D, Paw BH, Poss KD, et al.: Transplantation and in vivo imaging of multilineage engraftment in zebrafish bloodless mutants. Nat Immunol. 2003; 4(12): 1238-46.

PubMed Abstract | Publisher Full Text

van Ham TJ, Brady CA, Kalicharan RD, et al.: Intravital correlated microscopy reveals differential macrophage and microglial dynamics during resolution of neuroinflammation. Dis Model Mech. 2014; 7(7): 857-869.

PubMed Abstract | Publisher Full Text | Free Full Text

Vaz RL, Outeiro TF, Ferreira JJ: Zebrafish as an Animal Model for Drug

Discovery in Parkinson's Disease and Other Movement Disorders: A

Systematic Review. Front Neurol. 2018; 9: 347 .

PubMed Abstract | Publisher Full Text | Free Full Text

Veltkamp R, Gill D: Clinical Trials of Immunomodulation in Ischemic Stroke.

Neurotherapeutics. 2016; 13(4): 791-800.

PubMed Abstract | Publisher Full Text | Free Full Text

Walcott BP, Peterson RT: Zebrafish models of cerebrovascular disease. $J$ Cereb Blood Flow Metab. 2014; 34(4): 571-577.

PubMed Abstract | Publisher Full Text | Free Full Text
Wan S, Cheng Y, Jin H, et al.: Microglia Activation and Polarization After Intracerebral Hemorrhage in Mice: the Role of Protease-Activated Receptor-1. Trans/ Stroke Res. 2016; 7(6): 478-487.

PubMed Abstract | Publisher Full Text | Free Full Text

Wang J, Zhai W, Yu Z, et al.: Neuroprotection Exerted by Netrin-1 and Kinesin Motor KIF1A in Secondary Brain Injury following Experimental Intracerebral Hemorrhage in Rats. Front Cell Neurosci. 2018; 11: 432.

PubMed Abstract | Publisher Full Text | Free Full Text

Westerfield M: The zebrafish book: a guide for the laboratory use of zebrafish. 2000.

Reference Source

WHO: The top 10 causes of death [Online]. 2017; [Accessed 28/02/17].

Reference Source

Xi G, Keep RF, Hoff JT: Mechanisms of brain injury after intracerebral haemorrhage. Lancet Neurol. 2006; 5(1): 53-63.

PubMed Abstract | Publisher Full Text

Xue M, Del Bigio MR: Comparison of brain cell death and inflammatory reaction in three models of intracerebral hemorrhage in adult rats. $J$ Stroke Cerebrovasc Dis. 2003; 12(3): 152-159.

PubMed Abstract | Publisher Full Text

Yang J, Arima H, Wu G, et al:: Prognostic significance of perihematomal edema in acute intracerebral hemorrhage: pooled analysis from the intensive blood pressure reduction in acute cerebral hemorrhage trial studies. Stroke. 2015

46(4): 1009-13.

PubMed Abstract | Publisher Full Text

Yang J, Ding S, Huang W, et al:: Interleukin-4 Ameliorates the Functional Recovery of Intracerebral Hemorrhage Through the Alternative Activation of Microglia/Macrophage. Front Neurosci. 2016a; 10: 61.

PubMed Abstract | Publisher Full Text | Free Full Text

Yang SS, Lin L, Liu Y, et al.: High Morphologic Plasticity of Microglia/

Macrophages Following Experimental Intracerebral Hemorrhage in Rats. Int Mol Sci. 2016b; 17(7): pii: E1181.

PubMed Abstract | Publisher Full Text | Free Full Text

Yang R, Zhang Y, Huang D, et al:: Miconazole protects blood vessels from MMP9-dependent rupture and hemorrhage. Dis Model Mech. 2017; 10(3): 337-348. PubMed Abstract | Publisher Full Text | Free Full Text

Yeatts SD, Palesch YY, Moy CS, et al.: High dose deferoxamine in intracerebra hemorrhage (HI-DEF) trial: rationale, design, and methods. Neurocrit Care. 2013; 19(2): 257-266.

PubMed Abstract | Publisher Full Text | Free Full Text 


\title{
Open Peer Review
}

\section{Current Peer Review Status:}

Version 1

Reviewer Report 30 October 2018

https://doi.org/10.5256/f1000research.18006.r39224

(C) 2018 Zille M. This is an open access peer review report distributed under the terms of the Creative Commons Attribution License, which permits unrestricted use, distribution, and reproduction in any medium, provided the original work is properly cited.

\author{
Marietta Zille \\ 1 University of Lübeck, Institute for Experimental and Clinical Pharmacology and Toxicology, \\ Lübeck, Germany \\ 2 Fraunhofer Research Institution for Marine Biotechnology and Cell Technology, Lübeck, \\ Germany
}

In this manuscript, the authors propose two models of intracerebral hemorrhage (ICH) in zebrafish larvae to study post-hemorrhage brain injury. The authors compare a genetic (bubblehead) and chemically inducible (atorvastatin) model of spontaneous ICH and elegantly show assessment of cell death and inflammation in these models using live non-invasive imaging. In addition, they present data on locomotor activity as a functional outcome measurement.

First of all, I want to congratulate the authors on this high quality study that is timely and relevant to the ICH community as spontaneous models that allow higher throughput screening of potentially protective or regenerative compounds are clearly needed in the field (Hemorrhagic Stroke Academia Industry (HEADS) Roundtable Participants, 2018). Although new to the ICH community, zebrafish models have multiple advantages that may be of great use in this research area (as well as in others).

I have a couple of suggestions that I hope will be beneficial to further improve the quality of this excellent publication:

1. We and others have recently established that neurons die by multiple different regulated cell death mechanisms after ICH, including ferroptosis and necroptosis (Zille et al, 2017, Li et al, 2018). Therefore, the authors should discuss the limitation of the cell death marker, Annexin V, used in their study. Annexin V detects exposure of phosphatidiyl serine on the cell surface. On the one hand, this is not specific to apoptosis since when the cell membrane is disrupted phosphatidylserine can be detected intracellularly (and hence should not be claimed to analyze apoptotic cell death, e.g. in Methods section) (Munoz et al, 2013, Sawai et al, 2011, Zille et al, 2012). On the other hand, this only reflects a specific subset of cell death. This may also explain why cell death was detected only in a subset of larvae and at one specific time point which is also a relatively narrow time window. Is there a possibility to 
determine the involvement of other cell death mechanisms in zebrafish (e.g., GPX4 for ferroptosis or pRIP1/3 for necroptosis)? Undoubtedly, the exact cell death mechanisms warrant further exploration in future studies including pharmacological and molecular interventions.

2. The authors nicely show side-by-side comparison of both bubblehead and atorvastatin model. What is the main difference between the two? Therefore, the authors should make fluorescence pictures from both available in Fig. 2, the same time course in Fig. 3 as well as subgroup analysis in Fig. 4 and 5. Was only one of the two investigated regarding macrophages or are they pooled in this analysis?

3. One of the differences seems to be the occurrence of edema in the bubblehead model. This is very interesting as it is also a key feature in the ICH pathophysiology. The authors should provide pictures on both models over time. Is it possible to quantify the edema?

4. Locomotor activity: How does swimming time represent functional outcome of the larvae? Would distance travelled be an alternative readout?

5. What is the size of the hematoma and does it correlate with cell death, locomotor activity or macrophage activation?

6. For the atorvastatin model, was the $1 \mu \mathrm{M}$ dose used in Figure 2-5? If not, maybe the clusters of positive and negative for Annexin $V$ correspond to the dose of atorvastatin?

7. One of the major disadvantages of the larvae model is that it uses young animals. Is it possible to use atorvastatin to induce ICH in old zebrafish?

8. Regarding modeling human disease, another difference is that there is no mortality in this model at least not to the time points investigated. Do they die at earlier time points than their wildtype/untreated littermates? Do higher dosages of atorvastatin induce mortality in the larvae due to ICH?

Minor:

1. From the data where replicates were acquired, please specify whether the mean or median was chosen.

2. What is the \% MS222 used as lethal overdose?

Are a suitable application and appropriate end-users identified?

Yes

If applicable, is the statistical analysis and its interpretation appropriate?

Yes

Are the 3Rs implications of the work described accurately?

Yes

Is the rationale for developing the new method (or application) clearly explained?

Yes

Is the description of the method technically sound?

Yes

Are sufficient details provided to allow replication of the method development and its use by others?

Yes 
If any results are presented, are all the source data underlying the results available to ensure full reproducibility?

Yes

Are the conclusions about the method and its performance adequately supported by the findings presented in the article?

Yes

Competing Interests: No competing interests were disclosed.

Reviewer Expertise: Intracerebral haemorrhage, stroke researcher, cell death mechanisms

I confirm that I have read this submission and believe that I have an appropriate level of expertise to confirm that it is of an acceptable scientific standard, however I have significant reservations, as outlined above.

Author Response 02 Nov 2018

siobhan crilly

Dear Dr Zille,

Many thanks indeed for reviewing our manuscript and your helpful comments. Please see below for responses to your specific questions. Where necessary, we have made amendments in a second version of our manuscript, which we also refer to below.

We and others have recently established that neurons die by multiple different regulated cell death mechanisms after ICH, including ferroptosis and necroptosis (Zille et al, 2017, Li et al, 2018). Therefore, the authors should discuss the limitation of the cell death marker, Annexin $V$, used in their study. Annexin $V$ detects exposure of phosphatidiyl serine on the cell surface. On the one hand, this is not specific to apoptosis since when the cell membrane is disrupted phosphatidylserine can be detected intracellularly (and hence should not be claimed to analyze apoptotic cell death, e.g. in Methods section) (Munoz et al, 2013, Sawai et al, 2011, Zille et al, 2012). On the other hand, this only reflects a specific subset of cell death. This may also explain why cell death was detected only in a subset of larvae and at one specific time point which is also a relatively narrow time window. Is there a possibility to determine the involvement of other cell death mechanisms in zebrafish (e.g., GPX4 for ferroptosis or pRIP1/3 for necroptosis)? Undoubtedly, the exact cell death mechanisms warrant further exploration in future studies including pharmacological and molecular interventions.

We thank the reviewer for raising this point, and we appreciate that only using Annexin $V$ in this study limits the information that we can obtain about cell death mechanisms. As suggested, we have updated our discussion in version 2 to address this, and removed the term 'apoptosis' throughout. Of interest, in addition to the Annexin V reporter line, we have also verified cell death using an acridine orange assay in live larvae at the same time points and found an identical pattern of brain lesions in haemorrhaged animals only at $72 \mathrm{hpf}$ 
(data not shown). The primary purpose of this paper was to demonstrate that a range of 'translatable' disease outcomes exist following ICH in zebrafish larvae. However, our ongoing studies will aim to build a more accurate understanding of the precise mechanisms underlying brain injury following ICH in zebrafish larvae, which will include an investigation into other forms of cell death, such as necroptosis and ferroptosis.

\section{The authors nicely show side-by-side comparison of both bubblehead and atorvastatin model. What is the main difference between the two?}

Exposure to the HMG-CoA reductase inhibitor, atorvastatin (ATV) at 24 hours postfertilisation (hpf) leads to spontaneous cerebral-specific blood vessel rupture at $\sim 33 \mathrm{hpf}$ in zebrafish larvae at the onset of circulation (Eisa-Beygi et al., 2013, Shen et al., 2013, Huang et al., 2017, Li et al., 2017). By inhibiting HMG-CoA in neovascular neuroendothelial cells, ATV disrupts isoprenylation and hence activation of Rac1 (Xiao et al., 2013) and subsequent actin remodelling to stabilise VE cadherin mediated tight junctions (Eisa-Beygi and Rezaei, 2016). The result of this impairment is a 'leaky' cerebrovasculature and ICH between 3348hpf. Comparably, the 'bubblehead' (bbh) mutant line, which expresses a hypomorphic mutation in the arhgef7 gene, encoding the Rac GEF $\beta$ pix, also exhibit spontaneous ICH, as well as hydrocephalus, within a similar time frame to the ATV model (Liu et al., 2007, ten Klooster et al., 2006). Therefore it appears ICH is induced through comparable mechanistic defects in both ATV and bbh models. We have included a summary of this difference in new supplementary figure 1 in version 2 .

Therefore, the authors should make fluorescence pictures from both available in Fig. 2, the same time course in Fig. 3 as well as subgroup analysis in Fig. 4 and 5. Was only one of the two investigated regarding macrophages or are they pooled in this analysis?

In our updated version, we have now included images of the ATV model with Annexin $V$ cell death lesions at $3 \mathrm{dpf}$ in supplementary figure 2 . We have monitored locomotor function in the ATV model larvae over the same time course as figure 3, however the baseline measurements of control larvae at 3 and $4 \mathrm{dpf}$ was too low for a measurable outcome, and so we continued with the bbh mutant. We hypothesise this could be due to individual strain effect, as we have observed variable locomotor function in normal larvae between different strains. The ATV model was not used for the studies in figure 4 and 5 due to the antiinflammatory effects of statins (Bu et al., 2011).

One of the differences seems to be the occurrence of edema in the bubblehead model. This is very interesting as it is also a key feature in the ICH pathophysiology. The authors should provide pictures on both models over time. Is it possible to quantify the edema?

For the ATV model of ICH, larvae do not exhibit any oedema at any age. Also, although oedema occurs exclusively in $\mathrm{ICH}+$ bbh homozygotes it occurs in a stochastic fashion. Liu et al (2007) have shown that oedema and haemorrhage occur as separate entities in bbh mutants and develop independently of each other. For these reasons and the limitations raised in our discussion, we avoided using oedema as a pathological outcome for haemorrhagic stroke. 
Locomotor activity: How does swimming time represent functional outcome of the larvae? Would distance travelled be an alternative readout?

We have recorded distance travelled for both models in conjunction to time spent mobile and the trend is identical to that shown in Figure 3. We decided to use cumulative time spent mobile as this would be inclusive of seizure-like behaviour as well as normal thigmotactic behaviour (Turrini et al., 2017)

\section{What is the size of the hematoma and does it correlate with cell death, locomotor activity or macrophage activation?}

This is an extremely interesting question, and will form the basis of a future grant application. We have not accurately measured haematoma size, however we will use 3D rendering and light sheet microscopy to do so in the future. We are also interested in investigating haematoma location within the brain, to determine if this effects behavioural outcomes/specific neuronal populations.

For the atorvastatin model, was the $1 \mu \mathrm{M}$ dose used in Figure 2-5? If not, maybe the clusters of positive and negative for Annexin V correspond to the dose of atorvastatin?

Yes, we used 1 uM ATV, however we only performed ATV experiments in Figure 1-3, as discussed in methods and in response to your earlier question. Based on observation, we do not think ATV dosage affects severity of haemorrhage or haemorrhage-induced injury; rather it just affects the frequency of haemorrhage occurrence. We propose that once ICH occurs, the pathogenic response to blood in the brain is comparable between larvae, irrespective of ATV dosage.

\section{One of the major disadvantages of the larvae model is that it uses young animals. Is it} possible to use atorvastatin to induce ICH in old zebrafish?

We have used ATV to treat larvae at later time points to verify the findings in Eisa-Beygi et al (2013) and saw that haemorrhages were only induced when embryos are exposed to ATV prior to the critical neuroendothelial developmental time point at $\sim 36-48 \mathrm{hpf}$. Animals treated after this time do not haemorrhage.

We believe that the use of young animals is an advantage, especially given the potential for impacting the 3Rs. Furthermore as the animals age, pigmentation develops and the larvae are no longer transparent - therefore this significant experimental advantage will be lost using older animals. Admittedly, ICH is predominantly a disease associated with aging; however this condition does occur in children and young adults, making our model even more pertinent for understanding those types of presentation. Ultimately, however, our article does strongly indicate that ICH induces brain injury phenotypes in young zebrafish that are comparable to the aged human brain. Furthermore, through our observations, we know that zebrafish larvae recover from ICH at an astonishing rate and therefore offer a potentially powerful tool for studying resolution and recovery processes in the future.

Regarding modelling human disease, another difference is that there is no mortality in this model at least not to the time points investigated. Do they die at earlier time points than 


\section{their wildtype/untreated littermates?}

The bbh mutants do not die due to ICH. Indeed, these animals develop into healthy, fertile adults, which we propose is due to either developmental processes or the regenerate properties of zebrafish. Either way, understanding this recovery at the molecular/cellular level could provide essential insight into how we may one day recover the injured human brain.

\section{Do higher dosages of atorvastatin induce mortality in the larvae due to ICH?}

In the ATV model, high doses of ATV will kill the larvae; however we propose this is due to toxicity rather than ICH-related.

From the data where replicates were acquired, please specify whether the mean or median was chosen.

Mean was presented throughout. This has now been clarified in the latest version of the manuscript.

\section{What is the \% MS222 used as lethal overdose?}

4\% MS222 followed by maceration or freezing, which we have made clear in version 2.

Kind regards

Siobhan Crilly \& Paul Kasher

Competing Interests: none

Reviewer Report 24 October 2018

https://doi.org/10.5256/f1000research.18006.r39725

(C) 2018 Gibson C. This is an open access peer review report distributed under the terms of the Creative Commons Attribution License, which permits unrestricted use, distribution, and reproduction in any medium, provided the original work is properly cited.

\section{Claire L. Gibson}

University of Nottingham, Nottingham, UK

This article describes the development of a zebrafish model of intracerebral haemorrhage (ICH). ICH is a significant contributor to human mortality and morbidity and effect treatment options are limited. Research in this area has been hindered by the lack of appropriate models - although rodent models have contributed to our understanding of the pathology underlying ICH such models are based on a surgical intervention which does not mirror the spontaneous event, in 
humans, of ICH. Here the authors compare an existing genetic (bubblehead) and chemically inducible (atorvastatin) zebrafish models of spontaneous ICSH and importantly assess brain injury, locomotor function and neuroinflammation.

The article is well described with sufficient experimental detail and the authors clearly articulate the 3Rs benefits of such work. One of the particularly novel aspects of this work is the ability to image in vivo the $\mathrm{ICH}$-induced inflammatory processes and cellular interactions - this is not currently feasible in whole animal models, such as rodents. This may allow improved understanding of the early innate responses to spontaneous bleeding as occurs in ICH patients but which we cannot model in rodents.

Although the authors state that this paper used equipment and procedures that are commonplace and relatively simple to adopt in other labs it is worth mentioning what barriers need to be overcome in order to facilitate 'traditional' rodent researchers adopting such a model and species.

Specific comments:

Authors state that a proportion of ATV-treated embryos did not develop ICH and therefore were used a controls - what proportion was unsuccessful? Can the authors suggest why not all were successful? Did this result in a group of animals forming a control group which was treated separately to another control group (i.e. the untreated siblings)?

Not clear to me (although I am a rodent researcher!) how a brief exposure to anaesthesia at 72hpf would remove locomotor function bias or was necessary? Couldn't a baseline measurement be taken and data compared to this? The authors states that the exposure to this brief anaesthesia was at $72 \mathrm{hpf}$ but also state functional analyses were done at 72,96 and $120 \mathrm{hpf}$ - did the first time point occur coincidentally with the brief anaesthesia?

Authors have made available all underlying source data.

\section{Are a suitable application and appropriate end-users identified?}

Yes

If applicable, is the statistical analysis and its interpretation appropriate? Yes

Are the 3Rs implications of the work described accurately?

Yes

Is the rationale for developing the new method (or application) clearly explained? Yes

Is the description of the method technically sound?

Yes

Are sufficient details provided to allow replication of the method development and its use by others?

Yes 
If any results are presented, are all the source data underlying the results available to ensure full reproducibility?

Yes

Are the conclusions about the method and its performance adequately supported by the findings presented in the article?

Yes

Competing Interests: No competing interests were disclosed.

Reviewer Expertise: Referee suggested by the NC3Rs for their scientific expertise and experience in assessing 3Rs impact.

I confirm that I have read this submission and believe that I have an appropriate level of expertise to confirm that it is of an acceptable scientific standard.

Author Response 02 Nov 2018

siobhan crilly

Dear Prof Gibson,

Many thanks indeed for reviewing our manuscript and your helpful comments. Please see below for responses to your specific questions.

1. Authors state that a proportion of ATV-treated embryos did not develop ICH and therefore were used as controls - what proportion was unsuccessful? Can the authors suggest why not all were successful? Did this result in a group of animals forming a control group which was treated separately to another control group (i.e. the untreated siblings)? The ATV treated non-haemorrhaged group formed the ICH-control group for the ATV model. As you suggest, this group was separate from the untreated control group, and our subsequent analyses compared all 3 groups (i.e. untreated, $\mathrm{ICH}$ - and $\mathrm{ICH}+$ groups), as presented in Figure 2B and 3C. The proportion of ICH-controls varied with ATV dose, as presented in Figure 1B. For our cell death and motility assays, we used an ATV dosage of $1.0 \mu \mathrm{g} / \mathrm{ml}$, which resulted in approximately $20 \%$ of non-haemorrhaged animals, which we used as our ICH-group. We currently cannot explain why a proportion of ATV-treated larvae do not develop ICH. However as zebrafish are a relatively outbred model species, we hypothesise that a genetic component may explain increased neuroendothelial stability in some animals.

2. Not clear to me (although I am a rodent researcher!) how a brief exposure to anaesthesia at $72 \mathrm{hpf}$ would remove locomotor function bias or was necessary? Couldn't a baseline measurement be taken and data compared to this? The authors states that the exposure to this brief anaesthesia was at $72 \mathrm{hpf}$ but also state functional analyses were done at 72, 96 and $120 \mathrm{hpf}$ - did the first time point occur coincidentally with the brief anaesthesia?

Following fertilisation, we routinely plate $\mathrm{n}=50-100$ embryos per petri dish. At $72 \mathrm{hpf}$, natural 
variation in swimming behaviour/ability exists between individual larvae. For allocation to the locomotion assay, larvae are transferred from the petri dish into a 24 well plate using a pipette. To avoid always 'catching' the weakest/slowest swimmers, we briefly anaesthetise the animals so that larvae are randomly allocated to the assay. These fish were segregated at $72 \mathrm{hpf}$ for repeat assays at 96 and $120 \mathrm{hpf}$ and so there was no need to re-anaesthetise the animals for these later timepoints.

Kind regards

Siobhan Crilly \& Paul Kasher

Competing Interests: none

The benefits of publishing with F1000Research:

- Your article is published within days, with no editorial bias

- You can publish traditional articles, null/negative results, case reports, data notes and more

- The peer review process is transparent and collaborative

- Your article is indexed in PubMed after passing peer review

- Dedicated customer support at every stage

For pre-submission enquiries, contact research@f1000.com 